\title{
Genetic Programming IV
}




\title{
GENETIC PROGRAMMING SERIES
}

\author{
Series Editor \\ John Koza
}

Stanford University

Also in the series:

GENETIC PROGRAMMING AND DATA STRUCTURES: Genetic Programming + Data Structures $=$ Automatic Programming! William B. Langdon; ISBN: 0-7923-8135-1

AUTOMATIC RE-ENGINEERING OF SOFTWARE USING GENETIC PROGRAMMING, Conor Ryan; ISBN: 0-7923-8653-1

DATA MINING USING GRAMMAR BASED GENETIC PROGRAMMING AND APPLICATIONS, Man Leung Wong and Kwong Sak Leung; ISBN: 0-7923-7746-X

GRAMMATICAL EVOLUTION: Evolutionary Automatic Programming in an Arbitrary Language, Michael O'Neill and Conor Ryan; ISBN: 1-4020-7444-1 


\title{
Genetic Programming IV
}

\section{Routine Human-Competitive Machine Intelligence}

\author{
John R. Koza \\ Martin A. Keane \\ Matthew J. Streeter \\ William Mydlowec \\ Jessen Yu \\ Guido Lanza
}

缕 Springer 


\section{Library of Congress Cataloging-in-Publication Data}

Genetic programming IV : routine human-competitive intelligence / John R. Koza... [et al.] p. cm. (Genetic Programming series)

Includes bibliographical references and index.

ISBN 1-4020-7446-8 (hardcover) ISBN 0-387-25067-0 (paperback)

First paperback printing 2005

(C) 2003Springer Science+Business Media, Inc.

All rights reserved. This work may not be translated or copied in whole or in part without the written permission of the publisher (Springer Science+Business Media, Inc., 233 Spring Street, New York, NY 10013, USA), except for brief excerpts in connection with reviews or scholarly analysis. Use in connection with any form of information storage and retrieval, electronic adaptation, computer software, or by similar or dissimilar methodology now know or hereafter developed is forbidden.

The use in this publication of trade names, trademarks, service marks and similar terms, even if the are not identified as such, is not to be taken as an expression of opinion as to whether or not they are subject to proprietary rights.

Printed in the United States of America.

$\begin{array}{lllllllll}9 & 8 & 7 & 6 & 5 & 4 & 3 & 2 & 1\end{array}$

SPIN 11399841

springeronline.com 


\section{Advance Praise for \\ Genetic Programming IV: Routine Human-Competitive Machine Intelligence}

"In 1992, John Koza published his first book on genetic programming and forever changed the world of computation. At the time, many researchers, myself included, were skeptical about whether the idea of using genetic algorithms directly to evolve programs would ever amount to much. But scores of conquered problems and three additional books makes the case utterly persuasive. The latest contribution, Genetic Programming IV: Routine Human-Competitive Machine Intelligence, demonstrates the everyday solution of such 'holy grail' problems as the automatic synthesis of ana$\log$ circuits, the design of automatic controllers, and the automated programming of computers. This would be impressive enough, but the book also shows how to evolve whole families of solutions to entire classes of problems in a single run. Such parametric GP is a significant achievement, and I believe it foreshadows generalized evolution of complex contingencies as an everyday matter. To artificial evolutionaries of all stripes, I recommend that you read this book and breath in its thoughtful mechanism and careful empirical method. To specialists in any of the fields covered by this book's sample problem areas, I say read this book and discover the computeraugmented inventions that are your destiny. To remaining skeptics who doubt the inventive competence of genetics and evolution, I say read this book and change your mind or risk the strong possibility that your doubts will soon cause you significant intellectual embarrassment."

—David E. Goldberg, University of Illinois

"The research reported in this book is a tour de force. For the first time since the idea was bandied about in the 1940s and the early 1950s, we have a set of examples of human-competitive automatic programming."

— John H. Holland, University of Michigan

"The adaptive filters and neural networks that I have worked with over many years are self-optimizing systems where the relationship between performance (usually meansquare-error) and parameter settings (weights) is continuous. Optimization by gradient methods works well for these systems. Now, this book describes a wider class of optimization problems where the relationship between performance (fitness) and parameters is highly disjoint, and self-optimization is achieved by nature-inspired genetic algorithms involving random search (mutation) and crossover (sexual reproduction). John Koza and his colleagues have done remarkable work in advancing the development of genetic programming and applying this to practical problems such as electric circuit design and control system design. What is ingenious about their work is that they have found ways to approach design problems by parameterizing both physical and topological variables into a common code that can be subjected to genetic programming for optimization. It is amazing how this approach finds optimized solutions that are not obvious to the best human experts. This fine book gives an accounting of the latest work in genetic programming, and it is 'must reading' for 
those interested in adaptive and learning systems, neural networks, fuzzy systems, artificial intelligence, and neurobiology. I strongly recommend it.

-Bernard Widrow, Electrical Engineering Department, Stanford University

"John Koza's genetic programming approach to machine discovery can invent solutions to more complex specifications than any other I have seen."

—John McCarthy, Computer Science Department, Stanford University 
To our parents - all of whom were best-of-generation individuals 


\section{Acknowledgments}

Forrest H Bennett III, William Comisky, and Oscar Stiffelman participated in some of the work described in this book (as noted in the appropriate places throughout this book).

Frank Dunlap of Enabling Technology Inc. of Sunnyvale, California, made numerous helpful comments on the chapter on the post-2000 inventions.

Edgar Sheh of Western Digital Corporation made numerous helpful comments on the chapters on control.

David E. Goldberg of the University of Illinois at Urbana-Champaign, William Langdon of University College London, and Lee Spector of Hampshire College made extensive helpful suggestions concerning this book.

Kalyanmoy Deb of the Indian Institute of Technology at Kanpur; Adrian Stoica of JPL and NASA in Pasadena, California; and Stuart T. Smith of the University of North Carolina at Charlotte provided useful information for this book.

Jeffrey Schwarz of San Francisco, California, made numerous comments for improving earlier drafts of this book.

Douglas B. Kell of the University of Wales made comments on the description of our work on metabolic pathways.

We especially thank Ray Prill and Jon O. Satre of Gordon-Prill Inc. of Mountain View, California, for designing, constructing, and maintaining the site for our 1,000Pentium computer. 


\section{Biography of the Authors}

John R. Koza received his Ph.D. in Computer Science from the University of Michigan in 1972 under the supervision of John Holland. He was co-founder, Chairman, and CEO of Scientific Games Inc. from 1973 through 1987 where he co-invented the rub-off instant lottery ticket used by state lotteries. He has taught a course on genetic algorithms and genetic programming at Stanford University since 1988. He is currently a consulting professor in the Biomedical Informatics Program in the Department of Medicine at Stanford University and a consulting professor in the Department of Electrical Engineering at Stanford University.

John R. Koza [koza@Stanford.edu]

Martin A. Keane received a Ph.D. in Mathematics from Northwestern University in 1969. He worked for Applied Devices Corporation until 1972, in the Mathematics Department at General Motors Laboratory until 1976, and was Vice-President for Engineering of Bally Manufacturing Corporation until 1986. He is currently chief scientist of Econometrics Inc. of Chicago and a consultant to various computer-related and gaming-related companies.

Martin A. Keane [martinkeane@ ameritech.net]

Matthew J. Streeter received a Masters degree in Computer Science from Worcester Polytechnic Institute in 2001. His Masters thesis applied genetic programming to the automated discovery of numerical approximation formulae for functions and surfaces. His primary research interest is applying genetic programming to problems of real-world scientific or practical importance. He is currently working at Genetic Programming Inc. as a systems programmer and researcher.

Matthew J. Streeter [matt@genetic-programming.com]

William Mydlowec is Chief Executive Officer and co-founder of Pharmix Corporation, a venture-funded computational drug discovery company in Silicon Valley. He received his B.S. degree in Computer Science from Stanford University in 1998. He formerly did research at Genetic Programming Inc. with John Koza between 1997 and 2000.

William Mydlowec [bill@pharmix.com] 
Jessen Yu is Director of Engineering of Pharmix Corporation. He received a B.S. degree in Computer Science and Chemistry from Stanford University. He formerly did research at Genetic Programming Inc. with John Koza between 1998 and 2000.

Jessen Yu [jyu@pharmix.com]

Guido Lanza is Vice President of Biology and co-founder of Pharmix Corporation. He received his B.A. degree in 1998 from the University of California at Berkeley from the Department of Molecular and Cell Biology and Department of Integrative Biology. He received an M.Sc. in 1999 in Bioinformatics from the University of Manchester, UK. He formerly did research at Genetic Programming Inc. with John Koza in 2000 .

Guido Lanza [guido@Pharmix.com] 


\section{High-Level Table of Contents}

1 Introduction 1

2 Background on Genetic Programming 29

3 Automatic Synthesis of Controllers 49

4 Automatic Synthesis of Circuits 129

5 Automatic Synthesis of Circuit Topology, Sizing,
Placement, and Routing

6 Automatic Synthesis of Antennas 205

7 Automatic Synthesis of Genetic Networks 221

8 Automatic Synthesis of Metabolic Pathways 229

9 Automatic Synthesis of Parameterized Topologies for Controllers 281

10 Automatic Synthesis of Parameterized Topologies for Circuits 301

11 Automatic Synthesis of Parameterized Topologies with Conditional $\begin{array}{ll}\text { Developmental Operators for Circuits } & 341\end{array}$

12 Automatic Synthesis of Improved Tuning Rules for PID Controllers 367

13 Automatic Synthesis of Parameterized Topologies for $\begin{array}{ll}\text { Improved Controllers } & 387\end{array}$

14 Reinvention of Negative Feedback 413

15 Automated Reinvention of Six Post-2000 Patented Circuits 421

16 Problems for Which Genetic Programming May Be Well Suited 483

17 Parallel Implementation and Computer Time 515

18 Historical Perspective on Moore's Law and the Progression of Qualitatively More Substantial Results Produced by

Genetic Programming $\quad 523$

19 Conclusion $\quad 529$

Appendix A: Functions and Terminals $\quad 533$

Appendix B: Control Parameters $\quad 539$

Appendix C: Patented or Patentable Inventions Generated by
Genetic Programming

$\begin{array}{ll}\text { Bibliography } & 555\end{array}$ 


\section{Full Table of Contents}

1 Introduction 1

1.1 Genetic Programming Now Routinely Delivers High-Return

Human-Competitive Machine Intelligence 3

1.1.1 What We Mean by "Human-Competitive" 3

1.1.2 What We Mean by "High-Return" 4

1.1.3 What We Mean by "Routine" 5

1.1.4 What We Mean by "Machine Intelligence" 6

1.1.5 Human-Competitiveness of the Results Produced by

1.1.6 High-Return of the Results Produced by Genetic Programming 10

1.1.7 Routineness of the Results Produced by Genetic Programming 14

1.1.8 Machine Intelligence 15

1.2 Genetic Programming Is an Automated Invention Machine 15

1.2.1 The Illogical Nature of Invention and Evolution 19

1.2.2 Overcoming Established Beliefs 20

1.2.3 Automating the Invention Process 21

1.2.4 Patentable New Inventions Produced by Genetic Programming 22

1.3 Genetic Programming Can Automatically Create

$\begin{array}{ll}\text { Parameterized Topologies } & 23\end{array}$

1.4 Historical Progression of Qualitatively More Substantial Results

Produced by Genetic Programming in Synchrony with Increasing

Computer Power

2 Background on Genetic Programming 29

2.1 Preparatory Steps of Genetic Programming 29

2.2 Executional Steps of Genetic Programming 31

2.2.1 Example of a Run of Genetic Programming 34

2.3 Advanced Features of Genetic Programming 38

2.3.1 Constrained Syntactic Structures 38

2.3.2 Automatically Defined Functions $\quad 39$

2.3.3 Automatically Defined Iterations, Automatically Defined

Loops, Automatically Defined Recursions, and

Automatically Defined Stores 
2.3.4 Program Architecture and Architecture-Altering Operations $\quad 40$

2.3.5 Genetic Programming Problem Solver 41

2.3.6 Developmental Genetic Programming 41

2.3.7 Computer Code for Implementing Genetic Programming 42

2.4 Main Points of Four Books on Genetic Programming 42

2.5 Sources of Additional Information about Genetic Programming 45

3 Automatic Synthesis of Controllers 49

3.1 Background on Controllers 50

3.2 Design Considerations for Controllers 52

3.3 Representation of a Controller by a Block Diagram 53

3.4 Possible Techniques for Designing Controllers 58

3.4.1 Search by Hill Climbing $\quad 59$

3.4.2 Search by Gradient Methods $\quad 60$

3.4.3 Search by Simulated Annealing 61

3.4.4 Search by Genetic Algorithm and Genetic Programming 61

3.4.5 Previous Work on Controller Synthesis by Means of Genetic and Evolutionary Computation 62

3.4.6 Possible Approaches to Automatic Controller Synthesis Using Genetic Programming 62

3.5 Our Approach to the Automatic Synthesis of the Topology and Tuning of Controllers 64

3.5.1 Repertoire of Functions 65

3.5.2 Repertoire of Terminals 67

3.5.3 Representing the Plant 67

3.5.4 Automatically Defined Functions 68

3.5.5 Three Approaches for Establishing Numerical Parameter Values 69

3.5.5.1 Arithmetic-Performing Subtrees $\quad 70$

3.5.5.2 Perturbable Numerical Value 70

3.5.5.3 Arithmetic-Performing Subtree Containing Perturbable Numerical Values $\quad 71$

3.5.6 Constrained Syntactic Structure for Program Trees 73

3.6 Additional Representations of Controllers 73

3.6.1 Representation of a Controller by a Transfer Function 73

3.6.2 Representation of a Controller as a LISP Symbolic Expression 74

3.6.3 Representation of a Controller as a Program Tree 74

3.6.4 Representation of a Controller in Mathematica 75

3.6.5 Representation of a Controller and Plant as a Connection List 75

3.6.6 Representation of a Controller and Plant as a SPICE Netlist 78

$\begin{array}{lll}3.7 & \text { Two-Lag Plant } & 87\end{array}$

3.7.1 Preparatory Steps for the Two-Lag Plant 88

3.7.1.1 Program Architecture $\quad 88$

3.7.1.2 Terminal Set $\quad 88$

$\begin{array}{llr}3.7 .1 .3 & \text { Function Set } & 89\end{array}$

$\begin{array}{llr}3.7 .1 .4 & \text { Fitness Measure } & 89\end{array}$

3.7.1.5 Control Parameters 95 
3.7.1.6 Termination Criterion and Results Designation 95

3.7.1.7 Knowledge Incorporated into the Preparatory Steps 96

3.7.2 Results for the Two-Lag Plant 102

3.7.3 Human-Competitiveness of the Result for the Two-Lag Plant Problem

3.7.4 AI Ratio for the Two-Lag Plant Problem 112

$\begin{array}{lll}3.8 & \text { Three-Lag Plant } & 113\end{array}$

3.8.1 Preparatory Steps for the Three-Lag Plant 114

3.8.1.1 Program Architecture 114

$\begin{array}{lll}3.8 .1 .2 & \text { Terminal Set } & 114\end{array}$

3.8.1.3 Function Set $\quad 114$

3.8.1.4 Fitness Measure $\quad 114$

3.8.1.5 Control Parameters 115

3.8.2 Results for the Three-Lag Plant 115

3.8.3 Routineness for the Three-Lag Plant Problem 119

3.8.4 AI Ratio for the Three-Lag Plant Problem 119

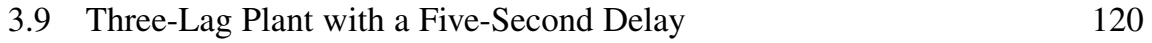

3.9.1 Preparatory Steps for the Three-Lag Plant with

$\begin{array}{ll}\text { 3.9.1.1 Program Architecture } & 120 \\ 3.9 .1 .2 & \text { Terminal Set }\end{array}$

3.9.1.2 Terminal Set 120

$\begin{array}{lll}3.9 .1 .3 & \text { Function Set } & 121\end{array}$

$\begin{array}{lll}3.9 .1 .4 & \text { Fitness Measure } & 121\end{array}$

3.9.1.5 Control Parameters 122

3.9.2 Results for the Three-Lag Plant with a Five-Second Delay 122

3.9.3 Routineness for the Three-Lag Plant with
a Five-Second Delay

3.9.4 AI Ratio for the Three-Lag Plant with a Five-Second Delay 123

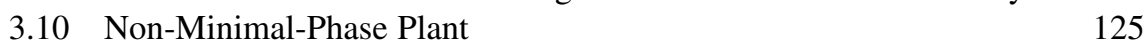

3.10.1 Preparatory Steps for the Non-Minimal-Phase Plant 125

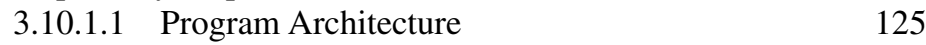

$\begin{array}{lll}3.10 .1 .2 & \text { Terminal Set } & 125\end{array}$

$\begin{array}{lll}3.10 .1 .3 & \text { Function Set } & 125\end{array}$

$\begin{array}{lll}3.10 .1 .4 & \text { Fitness Measure } & 125\end{array}$

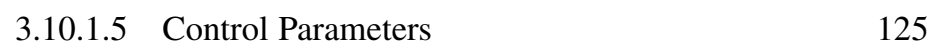

3.10.2 Results for the Non-Minimal Phase Plant 125

3.10.3 Routineness for the Non-Minimal Phase Plant Problem 127

3.10.4 AI Ratio for the Non-Minimal Phase Plant Problem 127

4 Automatic Synthesis of Circuits 129

4.1 Our Approach to the Automatic Synthesis of the Topology and

Sizing of Circuits 131

4.1.1 Evolvable Hardware 134

$\begin{array}{ll}\text { 4.2 Searching for the Impossible } & 135\end{array}$

4.2.1 Preparatory Steps for the RC Circuit with Gain Greater
than Two

$\begin{array}{lll}\text { 4.2.1.1 Initial Circuit } & 138\end{array}$


4.2.1.2 Program Architecture 139

4.2.1.3 Function Set 139

4.2.1.4 Terminal Set 140

4.2.1.5 Fitness Measure $\quad 141$

4.2.1.6 Control Parameters 142

4.2.2 Results for the RC Circuit with Gain Greater than Two 142

4.2.3 Routineness of the Transition from a Problem of Controller Synthesis to a Problem of Circuit Synthesis 143

4.2.4 AI Ratio for the RC Circuit with Gain Greater than Two 145

$\begin{array}{ll}\text { 4.3 Reinvention of the Philbrick Circuit } & 147\end{array}$

4.3.1 Preparatory Steps for the Philbrick Circuit 148

4.3.1.1 Initial Circuit 148

4.3.1.2 Program Architecture $\quad 149$

4.3.1.3 Terminal Set 149

4.3.1.4 Function Set 149

4.3.1.5 Fitness Measure 149

4.3.1.6 Control Parameters $\quad 150$

4.3.2 Results for the Philbrick Circuit 150

4.3.3 Human-Competitiveness of the Result for the Philbrick Circuit Problem 151

4.3.4 Routineness for the Philbrick Circuit Problem 152

4.3.5 AI Ratio for the Philbrick Circuit Problem 153

$\begin{array}{ll}\text { 4.4 Circuit for the NAND Function } & 153\end{array}$

4.4.1 Preparatory Steps for the NAND Circuit 154

4.4.1.1 Initial Circuit 154

4.4.1.2 Program Architecture $\quad 154$

4.4.1.3 Terminal Set 154

4.4.1.4 Function Set 154

4.4.1.5 Fitness Measure 156

4.4.1.6 Control Parameters 157

4.4.1.7 Termination Criterion $\quad 157$

4.4.2 Results for the NAND Circuit 157

4.4.3 Human-Competitiveness of the Result for the
NAND Circuit Problem

4.4.4 Routineness for the NAND Circuit Problem 159

4.4.5 AI Ratio for the NAND Circuit Problem 159

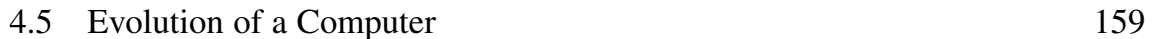

4.5.1 Preparatory Steps for the Arithmetic Logic Unit 160

$\begin{array}{lll}\text { 4.5.1.1 Initial Circuit } & 160\end{array}$

4.5.1.2 Fitness Measure 160

4.5.1.3 Control Parameters 161

4.5.2 Results for the Arithmetic Logic Unit 161

4.5.3 Routineness for the Arithmetic Logic Unit Circuit Problem 161

4.5.4 AI Ratio for the Arithmetic Logic Unit Circuit Problem 162

4.6 Square Root Circuit 162

4.6.1 Preparatory Steps for Square Root Circuit 163

4.6.1.1 Initial Circuit 163 
4.6.1.2 Program Architecture 163

4.6.1.3 Terminal Set 163

4.6.1.4 Function Set 163

4.6.1.5 Fitness Measure 163

4.6.1.6 Control Parameters $\quad 165$

4.6.2 Results for Square Root Circuit 165

4.6.3 Routineness for the Square Root Circuit Problem 168

4.6.4 AI Ratio for the Square Root Circuit Problem 168

4.7 Automatic Circuit Synthesis Without an Explicit Test Fixture 168

4.7.1 Preparatory Steps for the Lowpass Filter Problem Without an Explicit Test Fixture

4.7.1.1 Initial Circuit $\quad 169$

4.7.1.2 Program Architecture 171

4.7.1.3 Function Set 171

$\begin{array}{lll}\text { 4.7.1.4 Terminal Set } & 171\end{array}$

4.7.1.5 Fitness Measure $\quad 172$

4.7.1.6 Control Parameters 173

4.7.2 Results for the Lowpass Filter Problem without an Explicit Test Fixture

4.7.3 Routineness for the Lowpass Filter Problem without an Explicit Test Fixture

4.7.4 AI Ratio for the Lowpass Filter Problem without an Explicit Test Fixture

5 Automatic Synthesis of Circuit Topology, Sizing, Placement, and Routing 175

5.1 Our Approach to the Automatic Synthesis of Circuit Topology,

Sizing, Placement, and Routing

$\begin{array}{lll}\text { 5.1.1 Initial Circuit } & 177\end{array}$

$\begin{array}{ll}\text { 5.1.2 Circuit-Constructing Functions } & 178\end{array}$

$\begin{array}{ll}\text { 5.1.3 Component-Creating Functions } & 179\end{array}$

$\begin{array}{ll}\text { 5.1.4 Topology-Modifying Functions } & 181\end{array}$

5.1.5 Development-Controlling Functions 186

5.1.6 Developmental Process 186

5.2 Lowpass Filter with Layout 186

5.2.1 Preparatory Steps for the Lowpass Filter with Layout 186

5.2.1.1 Initial Circuit 186

5.2.1.2 Program Architecture $\quad 186$

5.2.1.3 Function Set 186

$\begin{array}{lll}5.2 .1 .4 & \text { Terminal Set } & 187\end{array}$

$\begin{array}{lll}\text { 5.2.1.5 Fitness Measure } & 187\end{array}$

5.2.1.6 Control Parameters 188

5.2.2 Results for the Lowpass Filter with Layout 188

5.2.3 Human-Competitiveness of the Result for the Lowpass Filter Problem with Layout

5.2.4 Routineness of the Transition from a Problem of Circuit Synthesis without Layout to a Problem of Circuit Synthesis with Layout 
5.2.5 AI Ratio for the Lowpass Filter Problem with Layout 197

$\begin{array}{lll}5.3 & 60 \mathrm{~dB} \text { Amplifier with Layout } & 197\end{array}$

5.3.1 Preparatory Steps for 60 dB Amplifier with Layout 197

$\begin{array}{lll}\text { 5.3.1.1 Initial Circuit } & 197\end{array}$

5.3.1.2 Program Architecture 197

5.3.1.3 Function Set 198

5.3.1.4 Terminal Set 198

5.3.1.5 Fitness Measure 198

5.3.1.6 Control Parameters 199

5.3.2 Results for 60 dB Amplifier with Layout 199

5.3.3 Routineness for the $60 \mathrm{~dB}$ Amplifier Problem with Layout 202

5.3.4 AI Ratio for the 60 dB Amplifier Problem with Layout 203

6 Automatic Synthesis of Antennas 205

6.1 Our Approach to the Automatic Synthesis of the Geometry and Sizing of Antennas 206

6.2 Illustrative Problem of Antenna Synthesis 207

6.3 Repertoire of Functions and Terminals 209

6.3.1 Repertoire of Functions 209

6.3.2 Repertoire of Terminals 210

6.3.3 Example of the Use of the Functions and Terminals 211

6.4 Preparatory Steps for the Antenna Problem 212

6.4.1 Program Architecture $\quad 212$

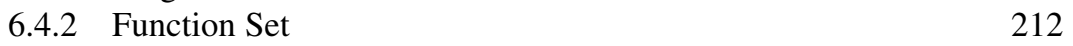

$\begin{array}{ll}\text { 6.4.3 Terminal Set } & 212\end{array}$

$\begin{array}{lll}6.4 .4 & \text { Fitness Measure } & 212\end{array}$

6.4.5 Control Parameters 216

6.5 Results for the Antenna Problem 216

6.6 Routineness of the Transition from Problems of

Synthesizing Controllers, Circuits, and Circuit Layout to

a Problem of Synthesizing an Antenna 219

6.7 AI Ratio for the Antenna Problem 220

7 Automatic Synthesis of Genetic Networks 221

7.1 Statement of the Illustrative Problem 221

7.2 Representation of Genetic Networks by Computer Programs 223

7.2.1 Repertoire of Functions 223

7.2.2 Repertoire of Terminals 224

$\begin{array}{lll}7.3 & \text { Preparatory Steps } & 224\end{array}$

7.3.1 Program Architecture 224

$\begin{array}{lll}\text { 7.3.2 Function Set } & 224\end{array}$

$\begin{array}{lll}7.3 .3 & \text { Terminal Set } & 224\end{array}$

7.3.4 Fitness Measure 224

$\begin{array}{ll}\text { 7.3.5 Control Parameters } & 225\end{array}$

$\begin{array}{lll}7.4 & \text { Results } & 225\end{array}$ 
7.4.1 Routineness of the Transition from Problems of Synthesizing Controllers, Circuits, Circuits With Layout, and Antennas to a Problem of Genetic Network Synthesis

7.4.2 AI Ratio for the Genetic Network Problem

8 Automatic Synthesis of Metabolic Pathways

8.1 Our Approach to the Automatic Synthesis of the Topology and Sizing of Networks of Chemical Reactions

8.2 Statement of Two Illustrative Problems

8.3 Types of Chemical Reactions 234

8.3.1 One-Substrate, One-Product Reaction 234

8.3.2 One-Substrate, Two-Product Reaction 243

8.3.3 Two-Substrate, One-Product Reaction 244

8.3.4 Two-Substrate, Two-Product Reaction 248

8.4 Representation of Networks of Chemical Reactions by

Computer Programs 250

8.4.1 Representation as a Program Tree 250

8.4.1.1 Repertoire of Functions in the Program Tree 250

8.4.1.2 Repertoire of Terminals 251

8.4.1.3 Constrained Syntactic Structure 251

8.4.1.4 Example of a Program Tree 251

8.4.2 Representation as a Symbolic Expression 255

8.4.3 Representation as a System of Nonlinear
Differential Equations

8.4.4 Representation as an Analog Electrical Circuit 259

8.4.5 Flexibility of the Representation 262

$\begin{array}{lll}8.5 & \text { Preparatory Steps } & 263\end{array}$

8.5.1 Program Architecture 263

8.5.2 Function Set 264

8.5.3 Terminal Set 264

8.5.4 Fitness Measure $\quad 264$

$\begin{array}{ll}\text { 8.5.5 Control Parameters } & 267\end{array}$

8.6 Results for the Phospholipid Cycle 267

8.6.1 Routineness of the Transition from Problems of

Synthesizing Controllers, Circuits, Circuits with Layout, Antennas, and Genetic Networks to a Problem of Synthesis of a Network of Chemical Reactions

8.6.2 AI Ratio for the Metabolic Pathway Problem for the Phospholipid Cycle 275

8.7 Results for the Synthesis and Degradation of Ketone Bodies 275

8.7.1 Routineness for the Metabolic Pathway Problem Involving Ketone Bodies 278

8.7.2 AI Ratio for the Metabolic Pathway Problem Involving Ketone Bodies $\quad 278$

$\begin{array}{lll}8.8 & \text { Future Work on Metabolic Pathways } & 278\end{array}$ 
8.8.1 Improved Program Tree Representation 278

$\begin{array}{lll}\text { 8.8.2 Null Enzyme } & 278\end{array}$

8.8.3 Minimum Amount of Data Needed 278

8.8.4 Opportunities to Use Knowledge 279

8.8.5 Designing Alternative Metabolisms 279

9 Automatic Synthesis of Parameterized Topologies for Controllers 281

9.1 Parameterized Controller for a Three-Lag Plant 282

9.1.1 Preparatory Steps for the Parameterized Controller for a Three-Lag Plant 283

9.1.1.1 Program Architecture 283

9.1.1.2 Terminal Set 283

9.1.1.3 Function Set 284

9.1.1.4 Fitness Measure $\quad 284$

9.1.1.5 Control Parameters 286

9.1.2 Results for the Parameterized Controller for a

9.1.3 Routineness of the Transition from a Problem

Involving a Non-Parameterized Controller to a Problem Involving a Parameterized Controller 290

9.1.4 AI Ratio for the Parameterized Controller for a Three-Lag Plant 291

9.2 Parameterized Controller for Two Families of Plants 291

9.2.1 Preparatory Steps for the Parameterized Controller for
Two Families of Plants

9.2.1.1 Program Architecture 292

9.2.1.2 Terminal Set 292

9.2.1.3 Function Set 293

9.2.1.4 Fitness Measure 293

9.2.1.5 Control Parameters 296

9.2.2 Results for the Parameterized Controller for

9.2.3 Human-Competitiveness of the Result for the Parameterized Controller for Two Families of Plants 299

9.2.4 Routineness for the Parameterized Controller for Two Families of Plants

9.2.5 AI Ratio for the Parameterized Controller for Two Families of Plants 300

10 Automatic Synthesis of Parameterized Topologies for Circuits 301

$\begin{array}{lll}10.1 & \text { Five New Techniques } & 301\end{array}$

10.1.1 New NODE Function for Connecting Distant Points 302

10.1.2 Symmetry-Breaking Procedure using Geometric Coordinates $\quad 303$

10.1.3 Depth-First Evaluation 304 
10.1.4 New TWO_LEAD Function for Inserting Two-Leaded Components

10.1.5 New Q Transistor-Creating Function 305

10.2 Zobel Network with Two Free Variables 306

10.2.1 Preparatory Steps for the Zobel Network Problem with Two Free Variables 307

10.2.1.1 Initial Circuit 307

10.2.1.2 Program Architecture 307

10.2.1.3 Function Set 307

10.2.1.4 Terminal Set 308

10.2.1.5 Fitness Measure $\quad 309$

10.2.1.6 Control Parameters 310

10.2.2 Results for the Zobel Network Problem with
Two Free Variables

10.2.3 Routineness of the Transition from a Problem Involving a Non-Parameterized Circuit to a Problem Involving a Parameterized Circuit

10.2.4 AI Ratio for the Zobel Network Problem with Two Free Variables

10.3 Third-Order Elliptic Lowpass Filter with a Free Variable for the Modular Angle

10.3.1 Preparatory Steps for the Third-Order Elliptic

Lowpass Filter with a Free Variable for the Modular Angle

10.3.1.1 Initial Circuit 313

10.3.1.2 Program Architecture $\quad 314$

10.3.1.3 Function Set 314

10.3.1.4 Terminal Set $\quad 314$

10.3.1.5 Fitness Measure $\quad 315$

10.3.1.6 Control Parameters 318

10.3.2 Results for the Lowpass Third-Order Elliptic Filter with a Free Variable for the Modular Angle 318

10.3.3 Routineness for the Lowpass Third-Order Elliptic Filter with a Free Variable for the Modular Angle

10.3.4 AI Ratio for the Lowpass Third-Order Elliptic Filter with a Free Variable for the Modular Angle

10.4 Passive Lowpass Filter with a Free Variable for

the Passband Boundary

10.4.1 Preparatory Steps for the Passive Lowpass Filter with a Free Variable for the Passband Boundary

10.4.1.2 Program Architecture $\quad 325$

10.4.1.3 Terminal Set 325

10.4.1.4 Function Set 326

10.4.1.5 Fitness Measure $\quad 326$

10.4.1.6 Control Parameters 328 
10.4.2 Results for the Passive Lowpass Filter with a Free Variable for the Passband Boundary

10.4.3 Routineness for the Passive Lowpass Filter with a Free Variable for the Passband Boundary

10.4.4 AI Ratio for the Passive Lowpass Filter with a Free Variable for the Passband Boundary

10.5 Active Lowpass Filter with a Free Variable for

the Passband Boundary

10.5.1 Preparatory Steps for the Active Lowpass Filter with a Free Variable for the Passband Boundary

10.5.1.1 Initial Circuit

333

10.5.1.2 Program Architecture

10.5.1.3 Function Set

10.5.1.4 Terminal Set

10.5.1.5 Fitness Measure

335

10.5.1.6 Control Parameters

10.5.2 Results for the Active Lowpass Filter with a Free Variable for the Passband Boundary

10.5.3 Routineness for the Active Lowpass Filter with a Free Variable for the Passband Boundary

10.5.4 AI Ratio for the Active Lowpass Filter with a Free Variable for the Passband Boundary

11 Automatic Synthesis of Parameterized Topologies with Conditional Developmental Operators for Circuits

11.1 Lowpass/Highpass Filter Circuit

11.1.1 Preparatory Steps for the Lowpass/Highpass Filter

11.1.1.1 Initial Circuit

11.1.1.2 Program Architecture

11.1.1.3 Terminal Set

11.1.1.4 Function Set

11.1.1.5 Fitness Measure

11.1.1.6 Control Parameters

11.1.2 Results for the Lowpass/Highpass Filter

11.1.3 Routineness of the Transition from a Parameterized

Topology Problem without Conditional Developmental

Operators to a Problem with Conditional Developmental Operators

11.1.4 AI Ratio for the Lowpass/Highpass Filter Problem 348

11.2 Lowpass/Highpass Filter with Variable Passband Boundary 348

11.2.1 Preparatory Steps for the Lowpass/Highpass Filter with Variable Passband Boundary

11.2.1.1 Fitness Measure

11.2.2 Results for the Lowpass/Highpass Filter with a Variable Passband Boundary 
11.2.3 Routineness for the Lowpass/Highpass Filter with a Variable Passband Boundary

11.2.4 AI Ratio for the Lowpass/Highpass Filter with a Variable Passband Boundary

11.3 Quadratic/Cubic Computational Circuit

11.3.1 Preparatory Steps for the Quadratic/

Cubic Computational Circuit $\quad 358$

11.3.1.1 Initial Circuit 358

11.3.1.2 Program Architecture $\quad 358$

11.3.1.3 Function Set $\quad 358$

11.3.1.4 Terminal Set $\quad 359$

$\begin{array}{lll}\text { 11.3.1.5 Fitness Measure } & 359\end{array}$

11.3.1.6 Control Parameters 360

11.3.2 Results for the Quadratic/Cubic Computational Circuit 360

$\begin{array}{lll}11.4 \text { A 40/60dB Amplifier } & 364\end{array}$

11.4.1 Preparatory Steps for the 40/60 dB Amplifier 364

11.4.1.1 Initial Circuit $\quad 365$

11.4.1.2 Terminal Set $\quad 365$

11.4.1.3 Fitness Measure $\quad 365$

11.4.1.4 Control Parameters $\quad 366$

11.4.2 Results for 40/60 dB Amplifier 366

12 Automatic Synthesis of Improved Tuning Rules for PID Controllers 367

$\begin{array}{lll}12.1 & \text { Test Bed of Plants } & 371\end{array}$

12.2 Preparatory Steps for Improved PID Tuning Rules 374

12.2.1 Program Architecture $\quad 375$

$\begin{array}{lll}12.2 .2 & \text { Terminal Set } & 375\end{array}$

$\begin{array}{lll}12.2 .3 & \text { Function Set } & 375\end{array}$

$\begin{array}{lll}\text { 12.2.4 Fitness Measure } & 375\end{array}$

12.2.5 Control Parameters 376

12.3 Results for Improved PID Tuning Rules 376

12.4 Human-Competitiveness of the Results for the Improved PID Tuning Rules

12.5 Routineness of the Transition from Problems Involving Parameterized Topologies for Controllers to a Problem Involving PID Tuning Rules 385

12.6 AI Ratio for the Improved PID Tuning Rules 385

13 Automatic Synthesis of Parameterized Topologies for $\begin{array}{ll}\text { Improved Controllers } & 387\end{array}$

13.1 Preparatory Steps for Improved General-Purpose Controllers 387 13.1.1 Function Set 388

13.1.2 Terminal Set 388

13.1.3 Program Architecture $\quad 389$

13.1.4 Fitness Measure $\quad 389$

13.1.5 Control Parameters 390 
13.2 Results for Improved General-Purpose Controllers

13.2.1 Results for First Run for Improved

General-Purpose Controllers

13.2.2 Results for Second Run for Improved General-Purpose Controllers

13.2.3 Results for Third Run for Improved General-Purpose Controllers

13.3 Human-Competitiveness of the Results for the Improved General-Purpose Controllers

13.4 Routineness for the Improved General-Purpose Controllers

13.5 AI Ratio for the Improved General-Purpose Controllers

14 Reinvention of Negative Feedback

14.1 Genetic Programming Takes a Ride on the Lackawanna Ferry 414

14.1.1 Fitness Measure

14.1.2 Initial Circuit, Function Set, Terminal Set, and Control Parameters

14.2 Results for the Problem of Reducing Amplifier Distortion

14.3 Human-Competitiveness of the Result for the Problem of Reducing Amplifier Distortion

14.4 Routineness for the Problem of Reducing Amplifier Distortion

14.5 AI Ratio for the Problem of Reducing Amplifier Distortion

15 Automated Reinvention of Six Post-2000 Patented Circuits

15.1 The Six Circuits

15.1.1 Low-Voltage Balun Circuit

15.1.2 Mixed Analog-Digital Variable Capacitor 423

15.1.3 Voltage-Current Conversion Circuit 423

15.1.4 Low-Voltage High-Current Transistor Circuit 424

15.1.5 Cubic Function Generator $\quad 424$

15.1.6 Tunable Integrated Active Filter 426

15.2 Uniformity of Treatment of the Six Problems 426

15.3 Preparatory Steps for the Six Post-2000 Patented Circuits 428

15.3.1 Initial Circuit 428

15.3.1.1 Test Fixture for the Low Voltage Balun Circuit 428

15.3.1.2 Test Fixture for the Mixed Analog-Digital Variable Capacitor Circuit 429

15.3.1.3 Test Fixture for High-Current Load Circuit 430

15.3.1.4 Test Fixture for the Voltage-Current Conversion Circuit 431

15.3.1.5 Test Fixture for the Cubic Function Generator 432

15.3.1.6 Test Fixture for the Tunable Integrated Active Filter 432

15.3.2 Program Architecture 433

15.3.3 Function Set 433

15.3.4 Terminal Set $\quad 434$ 
15.3.5 Fitness Measure 435

15.3.5.1 Fitness Measure for Low Voltage Balun Circuit 436

15.3.5.2 Fitness Measure for Mixed Analog-Digital Variable Capacitor $\quad 437$

15.3.5.3 Fitness Measure for High-Current Load Circuit 440

15.3.5.4 Fitness Measure for Voltage-Current Conversion Circuit 441

15.3.5.5 Fitness Measure for Cubic Function Generator 442

15.3.5.6 Fitness Measure for Tunable Integrated Active Filter $\quad 442$

15.3.6 Control Parameters $\quad 444$

15.4 Results for the Six Post-2000 Patented Circuits 444

15.4.1 Results for Low-Voltage Balun Circuit 444

15.4.2 Results for Mixed Analog-Digital Variable Capacitor 451

15.4.3 Results for High-Current Load Circuit 454

15.4.3.1 Results for First Run of High-Current Load Circuit

15.4.3.2 Results for Second Run of High-Current Load Circuit

15.4.4 Results for Voltage-Current Conversion Circuit 458

15.4.5 Results for Cubic Function Generator 461

15.4.5.1 Results for First Run of Cubic

Function Generator

15.4.5.2 Results for Second Run of Cubic Function Generator $\quad 464$

15.4.6 Tunable Integrated Active Filter 466

15.4.6.1 Results for First Run of Tunable Integrated Active Filter

15.4.6.2 Results for Second Run of Tunable Integrated Active Filter

15.4.6.3 Results of Third Run of Tunable Integrated Active Filter

15.4.6.4 Results of Fourth Run of Tunable Integrated Active Filter

15.5 Commercial Practicality of Genetic Programming for Automated Circuit Synthesis

15.6 Human-Competitiveness of the Results for the Six Post-2000 Patented Circuits

15.7 Routineness for the Six Post-2000 Patented Circuits 481

15.8 AI Ratio for the Six Post-2000 Patented Circuits 482

16 Problems for Which Genetic Programming May Be Well Suited 483

16.1 Characteristics Suggesting the Use of the Genetic Algorithm 483

16.2 Characteristics Suggesting the Use of Genetic Programming 484

16.2.1 Discovering the Size and Shape of the Solution 484

16.2.2 Reuse of Substructures 486

16.2.3 The Number of Substructures 495 
16.2.4 Hierarchical References among the Substructures 496

16.2.5 Passing Parameters to Substructures 497

16.2.6 Type of Substructures 499

16.2.7 Number of Arguments Possessed by Substructures $\quad 500$

16.2.8 The Developmental Process 500

16.2.9 Parameterized Topologies Containing Free Variables 504

16.3 Characteristics Suggesting the Use of Genetic Methods 505

16.3.1 Non-Greedy Nature of Genetic Methods 505

16.3.2 Recombination in Conjunction with the Population in Genetic Methods 506

16.3.2.1 The Changing Mix of Schemata 507

16.3.2.2 Viewing Mutation and Crossover in a Common Framework 510

16.3.2.3 Taking Advantage of the Information Stored in the Population in Allocating Future Trials 511

17 Parallel Implementation and Computer Time 515

17.1 Computer Systems Used for Work in This Book 516

17.1.1 Alpha Parallel Computer System 516

17.1.2 Pentium Parallel Computer System $\quad 517$

17.2 Computer Time for Problems in This Book 518

18 Historical Perspective on Moore's Law and the Progression of Qualitatively More Substantial Results Produced by

Genetic Programming

18.1 Five Computer Systems Used in 15-Year Period 523

18.2 Qualitative Nature of Results Produced by the Five Computer Systems

18.3 Effect of Order-of-Magnitude Increases in Computer Power on the Qualitative Nature of the Results Produced by Genetic Programming

19 Conclusion

19.1 Genetic Programming Now Routinely Delivers High-Return Human-Competitive Machine Intelligence

19.2 Genetic Programming Is an Automated Invention Machine 530

19.3 Genetic Programming Can Automatically Create Parameterized Topologies

19.4 Genetic Programming Has Delivered Qualitatively More

Substantial Results in Synchrony with Increasing

Computer Power

Appendix A: Functions and Terminals 533

Appendix B: Control Parameters $\quad 539$

Appendix C: Patented or Patentable Inventions Generated by 